\title{
Downregulation of nucleoporin 88 and 214 induced by oridonin may protect OCIM2 acute erythroleukemia cells from apoptosis through regulation of nucleocytoplasmic transport of NF-кB
}

\author{
SHA YI ${ }^{*}$, YAN CHEN ${ }^{*}$, LU WEN, LIJING YANG and GUOHUI CUI \\ Department of Hematology, Union Hospital, Tongji Medical College, Huazhong University \\ of Science and Technology, Wuhan, Hubei, P.R. China
}

Received April 28, 2012; Accepted June 19, 2012

DOI: $10.3892 /$ ijmm.2012.1067

\begin{abstract}
Oridonin has been utilized for the treatment of various human diseases due to its anti-inflammatory, antibacterial and antitumor effects. However, the precise mechanisms induced by oridonin in human erythroleukemia are yet to be clarified. The present study aimed to elucidate possible oridonin-induced apoptotic mechanisms in OCIM2 cells, as well as the possible mechanisms whereby OCIM2 cells are relatively resistant to oridonin. Results in the present study showed that oridonin significantly inhibited OCIM2 and OCI-AML3 cell proliferation in a dose- and time-dependent manner, while expressing a much higher $\mathrm{IC}_{50}$ in OCIM2, compared to OCI-AML3. These results also indicated that oridonin induced OCIM2 cell apoptosis, associated with p65 and Bax, while activating caspases-9, -6 and -3 . However, p65 was only partly translocated into the nucleus, while most of the p65 was retained in the cytoplasm. Moreover, nucleoporin 214 (Nup214) and nucleoporin 88 (Nup88) were downregulated at the transcriptional and protein levels, subsequent to oridonin treatment. Taken together, these data suggest that oridonin has the potential to induce OCIM2 cell-apoptosis, involving $\mathrm{NF}-\kappa \mathrm{B}$ activation, whereas the downregulation of Nup88 and Nup214 may protect OCIM2 through the regulation of the nucleocytoplasmic transport of p65.
\end{abstract}

\section{Introduction}

AML is an aggressive malignancy distinguished from normal hematopoietic cells by key properties, including blocked differ-

Correspondence to: Professor Guohui Cui, Department of Hematology, Union Hospital, Tongji Medical College, Huazhong University of Science and Technology, Wuhan, Hubei, P.R. China

E-mail: ghcui@medmail.com.cn

*Contributed equally

Key words: oridonin, acute erythroleukemia, nucleoporin 88/214, $\mathrm{NF}-\kappa \mathrm{B}$, apoptosis entiation, enhanced self-renewal and increased proliferation. Although AML treatment is among the most dose-intensive approaches in the clinic, AML-related mortality remains high. Acute erythroleukemia is an extremely rare subtype of AML, associated with an extremely poor response to currently available therapeutic agents as well as survival rate, compared to other subtypes (1). Given the difficulties in well-tolerance treatment, novel chemotherapeutic agents and more effective therapies are urgently needed for the treatment of acute erythroleukemia.

$\mathrm{NF}-\kappa \mathrm{B}$, a member of the transcription factors family, is widely expressed in almost every cell, while being crucial in several signal transduction pathways (2). A common form of $\mathrm{NF}-\kappa \mathrm{B}$ is a heterodimer, consisting of p65 (RelA) and $\mathrm{p} 50$, present in the cytoplasm of unstimulated cells as an inactive I $\kappa \mathrm{B} \alpha$-bound formation. It may act both as a pro- and an anti-apoptotic transcription factor through the regulation of different target genes, such as Bcl-2, Bcl-xL, JNK kinase and p53 (2,3). Although NF- $\kappa \mathrm{B}$ activation is often associated with increased survival of cancer cells and resistance to chemotherapy, there is also evidence that NF- $\kappa \mathrm{B}$ activation sensitizes cells to apoptosis $(4,5)$.

Nucleoporin 88 (Nup88) is a non-FG nucleoporin found exclusively on the cytoplasmic face of the nuclear pore complex (NPC) that mediates nucleocytoplasmic trafficking of macromolecules (6). In tumors, Nup88 staining is prominent in the cytoplasm, often in granular dots $(7,8)$. Furthermore, its expression levels are highly correlated with the metastasis and mortality rates of colon cancer (9). Additionally, Nup88 is reported to be closely associated with CAN/Nup214, another nucleoporin and a proto-oncogene involved in leukemia (10). A study carried out on Drosophila reported that Drosophila larvae lacking either Nup214 or Nup88 showed a notable cytoplasmic accumulation of the NF- $\kappa$ B homologs (the NES-containing Dorsal and Dif) that normally shuttle between the nucleus and the cytoplasm. The FG repetition domain of Nup214 as well as the $\mathrm{N}$-terminal $\beta$-propeller domain of Nup88 bind directly to CRM-1/exportin-1, the receptor exporting most proteins from the nucleus.

In the present study, the effects of oridonin on OCIM2 cells were investigated. OCIM2 was found to be relatively resistant to oridonin treatment. Oridonin inhibited OCIM2 
growth and induced OCIM2 cell apoptosis by upregulating p65 and its target gene Bax and activating apoptosis executioners including caspases- $6,-9$ and -3 , while the downregulation of Nup214 and Nup88 may protect OCIM2 by regulating the nucleocytoplasmic transport of $\mathrm{p} 65$.

\section{Materials and methods}

Reagents. Oridonin of more than $98 \%$ purity (empirical formula, $\mathrm{C}_{20} \mathrm{H}_{28} \mathrm{O}_{6}$; molecular weight, 360.42), purchased from Sigma-Aldrich (St. Louis, MO, USA), was initially dissolved in dimethyl sulfoxide (DMSO), stored at $-20^{\circ} \mathrm{C}$ and thawed before use (Fig. 1A). RPMI-1640 medium was purchased from Gibco-BRL (Gaithersburg, MD, USA). Fetal bovine serum (FBS) was purchased from Shijiqing (HangZhou, China). DMSO, trypan blue, Hoechst 33258 and 3-(4,5-dimethylthiazol-2-yl)-2,5-diphenyltetrazoliumbromide (MTT) were purchased from Sigma-Aldrich. RNA TRIzol reagents were purchased from Invitrogen Life Technologies (Carlsbad, CA, USA) and the RT-PCR kit was obtained from Toyobo Biologics (Osaka, Japan). Primary antibody caspases-3, -6 and Bax were purchased from Cell Signaling Technology (Danvers, MA, USA). Primary antibody of Nup88 was purchased from Santa Cruz Biotechnology, Inc. (Santa Cruz, CA, USA). Primary antibodies of p65 and caspase-9 were purchased from Proteintech Group (Chicago, IL, USA). Nup214 was purchased from Abcam (Abcam, San Francisco, CA, USA). Horseradish peroxidase (HRP)-labeled secondary antibodies and $\gamma$-tubulin antibody were purchased from Jackson ImmunoResearch (Jackson, WY, USA). ECL western blotting detection reagents and analysis system was purchased from Pierce Biotechnology, Inc. (Rockford, IL, USA).

Cell lines and culture. The OCI-AML3 and OCIM2 were kindly provided by MD Minden (Ontario Cancer Institute, Toronto, ON, Canada). OCI/AML3 (FAB M4) was obtained from a patient with NPM mutation and OCIM2 (FAB-M6) from a patient with erythroleukemia. The cells were grown in RPMI-1640 and supplemented with 10\% FBS. Cultures were maintained in a humidified atmosphere with $5 \% \mathrm{CO}_{2}$ at $37^{\circ} \mathrm{C}$.

Cell proliferation assay. The anti-proliferative effect of oridonin on the OCIM2 and OCI-AML3 cells was determined using MTT assay, according to the Mosmann method. The OCIM2 and OCI-AML3 cells ( $1 \times 10^{4} /$ well) were exposed to varying concentrations of oridonin $(0-32 \mu \mathrm{mol} / \mathrm{l})$ for 24,48 and $72 \mathrm{~h}$ on 96 -well plates. Thereafter, $20 \mu \mathrm{l}$ MTT solutions ( $5 \mathrm{mg} / \mathrm{ml}$ in PBS) were added to each well. Subsequent to 4-h incubation, the supernatant was discarded and $150 \mu \mathrm{l}$ of DMSO was added. Once the blue crystals were dissolved, the optical density (OD) was measured at $490 \mathrm{~nm}$ using a 96-well multiscanner autoreader (BioTek Instruments, Winooski, VT, USA). The inhibition of cell proliferation was determined using the formula: inhibition of cell proliferation $(\%)=[1-\mathrm{OD}$ of the experimental samples/OD of the control)] $\mathrm{x} 100 \%$ ( $\mathrm{n}=3$, mean $\pm \mathrm{SD}$ ). The half maximal inhibitory concentration $\left(\mathrm{IC}_{50}\right)$ was defined as the oridonin concentration that causes $50 \%$ inhibition of cell proliferation.
Trypan blue dye exclusion assay. To assess the cytotoxicity of oridonin on OCIM2, the cells were treated with varying concentrations of oridonin $(0,8,16$ and $32 \mu \mathrm{mol} / \mathrm{l})$ for 12 , 24, 36 and $48 \mathrm{~h}$, and were then stained using the Trypan Blue Staining Cell Viability Assay (Beyotime, Haimen, China). Trypan blue staining was evaluated under the microscope and cells were counted using a hemocytometer. Cells that took up trypan blue were counted as dead cells.

Flow cytometry for apoptosis. Immunofluorescence flow cytometry was employed to evaluate the apoptosis-inducing effect of oridonin on the OCIM2 cells. The cells seeded on 6 -well plates $\left(5 \times 10^{5} / \mathrm{ml}\right)$ were treated with varying concentrations of oridonin $(0,2,8$ and $16 \mu \mathrm{mol} / \mathrm{l})$ for $24 \mathrm{~h}$. The cells were harvested and washed with cold PBS, and then resuspended in $100 \mu \mathrm{l}$ binding buffer. Subsequently, phosphatidyl serine was detected on the surface of apoptotic cells using Annexin V/ FITC and a PI apoptosis detection kit, following the manufacturer's instructions (Bender MedSystems, Inc., Burlingame, CA, USA). The number of cells in apoptosis was evaluated by FACS flow cytometry (BD Biosciences, San Diego, CA, USA). A population of at least 10,000 cells was analyzed.

Hoechst 33258 staining assay. Nuclear fragmentation was visualized by Hoechst 33258 staining of apoptotic nuclei. Cells treated with varying concentrations of oridonin $(0,2,8$ and $16 \mu \mathrm{mol} / \mathrm{l})$ for $24 \mathrm{~h}$ were collected and washed, and then fixed for $10 \mathrm{~min}$ prior to deposition onto polylysine-coated coverslips. Subsequently, the samples were permeabilized with $0.25 \%$ Triton X-100 for $5 \mathrm{~min}$ and stained with $1 \mu \mathrm{g} / \mathrm{ml}$ Hoechst 33258 for $30 \mathrm{~min}$ at $37^{\circ} \mathrm{C}$. The slides were mounted with glycerol-PBS, and the images were visualized and captured using a FV500 confocal microscope (Olympus, Tokyo, Japan).

Western blot analysis. Western blot analysis was used to evaluate the levels of Bax, caspases-3, -6, -9, Nup88, Nup214 and p65 in the OCIM2 cells exposed to oridonin. The OCIM2 cells $\left(1 \times 10^{6}\right)$ were seeded and treated in vitro with different concentrations of oridonin $(0,2,8$ and $16 \mu \mathrm{mol} / \mathrm{l})$ for $24 \mathrm{~h}$. Cells were lysed in RIPA buffer $(50 \mathrm{mM}$ Tris-HCl, $\mathrm{pH} 7.5$; $150 \mathrm{mM} \mathrm{NaCl} ; 1 \% \mathrm{NP}-40 ; 0.5 \%$ sodium deoxycholate; $0.1 \%$ SDS) containing complete protease inhibitor cocktail (Calbiochem, San Diego, USA). Nuclear and cytosolic extracts were prepared according to the manufacturer's instructions (Beyotime). The sample lysates ( $80 \mu \mathrm{g}$ of protein/ lane) were prepared, and the proteins were separated by $10 \%$ polyacrylamide gel. The proteins were electro-transferred onto nitrocellulose (NC) membranes and the blots were incubated with a blocking solution (5\% skimmed milk in Trisbuffered saline Tween-20 buffer) for $2 \mathrm{~h}$ at room temperature. The membranes were then incubated using the dilutions (1:500-1:1,000) of the primary antibodies. The primary antibodies were washed and incubated with secondary antibodies for $2 \mathrm{~h}$ at room temperature. The bound antibodies were visualized using a supersignal chemiluminescence detection (ECL) kit (Pierce Biotechnology, Inc., Rockford, IL, USA). The signals were detected using a chemiluminescence detection system (Bio-Rad, Hercules, CA, USA). The results were expressed as the relative density of the protein normalized to 
A

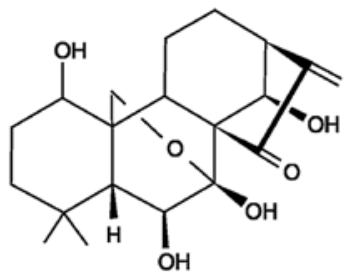

Oridonin

C

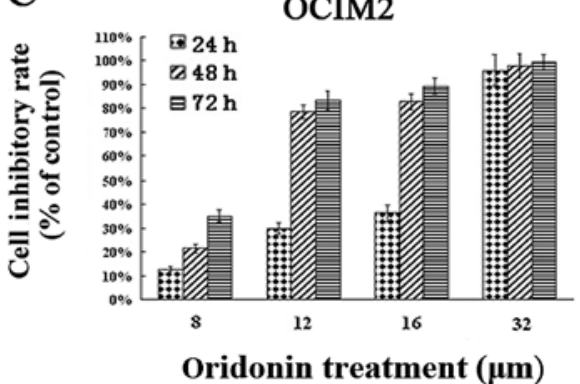

B

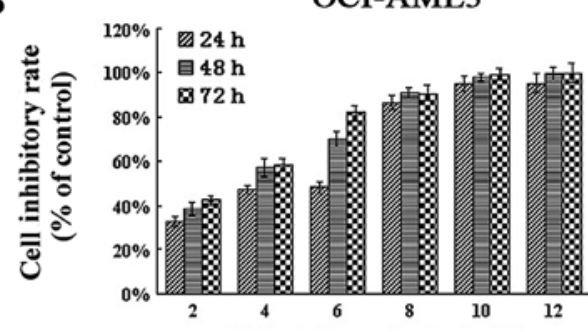

Oridonin treatment $(\mu \mathrm{m})$

D

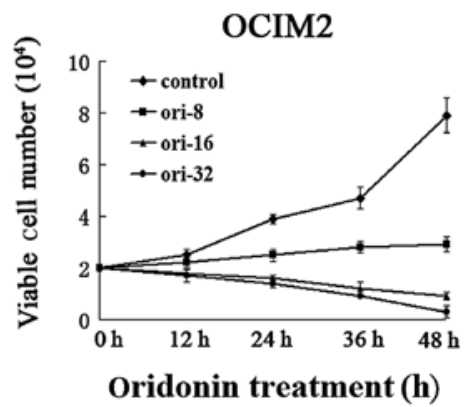

Figure 1. Effect of oridonin on the proliferation and vitality of acute myeloid leukemia cells. Oridonin inhibits the vitality and proliferation of human acute myeloid leukemia cells. (A) Chemical structure of oridonin. (B) OCI-AML3 and (C) OCIM2 were treated with indicated doses of oridonin for 24, 48 and $72 \mathrm{~h}$, and the viable cells were measured with MTT assay. (D) OCIM2 cells were treated with increasing doses of oridonin $(0,2,8$ and $16 \mu \mathrm{mol} / \mathrm{l})$ at the indicated time points $(12,24,36$ and $48 \mathrm{~h})$ and the vitality was detected by trypan blue stain exclusion assay. Data are presented as the mean \pm SD from three independent experiments.

$\gamma$-tubulin. Enhancement or inhibition rates were estimated by comparison with the control (100\%).

Quantitative real-time RT-PCR analysis. Total RNA was isolated from each group of OCIM2 cells treated with $0,2,8$ and $16 \mu \mathrm{mol} / \mathrm{l}$ oridonin for $24 \mathrm{~h}$, according to the manufacturer's instructions. Total RNA $(2 \mu \mathrm{g})$ was reverse transcribed into cDNA, according to the manufacturer's instructions using the TOYOBO kits. The analysis was carried out using SYBRGreen PCR master mix. Real-time RT-PCR amplification was performed in an ABI Prism 7900HT Sequence Detection System (Applied Biosystems, Foster City, CA, USA). Samples were run in triplicates in three independent experiments. All the threshold cycle (CT) values were normalized with $\beta$-actin in the same master reaction. The $2^{-\Delta \Delta \mathrm{CT}}(\Delta \Delta$ threshold cycle) method of analysis was used to relatively quantify the transcriptional levels of studied genes. Specific primers were used for human Nup88, forward: 5'-GGA GCT TGC TTT GAA ACT GG-3' and reverse: 5'-ATT TCC CGC AGA CTT TTC CT-3'; Nup214, forward: 5'-AGT CCT CAG TCT TGC CCT CA-3' and reverse: 5'-CGA TTG TTG GCT AGG GTG TT-3'; $\beta$-actin, forward: 5'-GCC CAG TCC TCT CCC AAG TC-3' and reverse: 5'-GGC ACG AAG GTC ATC ATT C-3'.

Immunofluorescence staining. An immunofluorescence staining assay was performed to evaluate the oridonin-induced enhancement and translocation effect of p65 on OCIM2 cells. The OCIM2 cells were exposed to varying concentrations of oridonin. After $24 \mathrm{~h}$, the cells were fixed with $4 \%$ paraformaldehyde for $10 \mathrm{~min}$ and permeabilized with $0.25 \%$ Triton X-100 for $5 \mathrm{~min}$. The cells were then washed twice with PBS, blocked in $3 \%$ bovine serum albumin and then deposited onto polylysine coated coverslips. The rabbit polyclonal anti-p65 antibody was diluted to 1:100 and non-immunoreactive IgG was added as the negative control. Subsequent to washing, the cells were incubated with Cy3-labeled goat anti-rabbit secondary antibody (Pierce Biotechnology, Rockford, IL, USA), diluted in PBS for $2 \mathrm{~h}$ and stained with Hoechst 33258 $(10 \mu \mathrm{g} / \mathrm{ml})$ for $10 \mathrm{~min}$. The image was visualized and captured using a FV500 confocal microscope (Olympus). The p65 levels were estimated as the mean fluorescence intensity after subtracting those of the negative control cells.

Statistical analysis. Data were presented as the mean \pm SD of at least three independent experiments. The t-tests were used to determine the differences between the treated samples and the controls. $\mathrm{P}<0.05$ was considered to indicate a statistically significant difference.

\section{Results}

Effects of oridonin on the proliferation and growth of $A M L$ cells. Initially, the effect of oridonin on the proliferation of AML cells was examined. OCI-AML3 and OCIM2 were cultured in varying concentrations of oridonin, while the inhibitory effects of oridonin were examined by MTT, indicating the mitochondrial activity of the cells. Results in Fig. 1 show that oridonin had a dose- and time-dependent anti-proliferative effect on the OCI-AML3 (Fig. 1B), as well as on the OCIM2 cells (Fig. 1C), subsequent to 24-, 48- and 72-h exposure. The number of viable cells decreased with the increasing concentration of oridonin over the exposure. The $\mathrm{IC}_{50}$ value of OCIM2 at $24 \mathrm{~h}$ was $15.07 \pm 1.34 \mu \mathrm{mol} / 1$, while that of OCI-AML3 was only $3.27 \pm 0.23 \mu \mathrm{mol} / \mathrm{l}$. As 
A

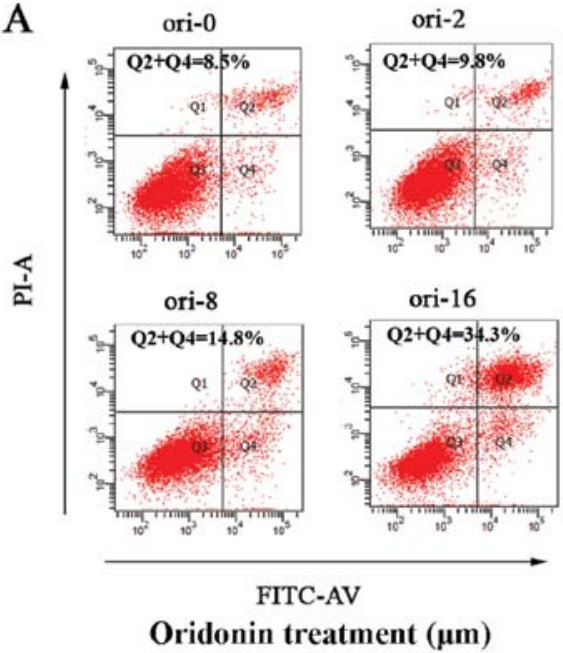

B

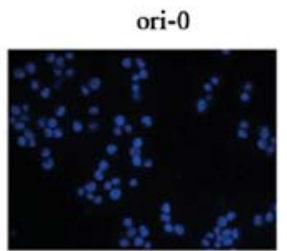

ori-8

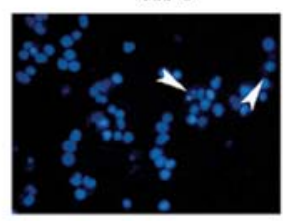

Oridonin treatment $(\mu \mathrm{m})$

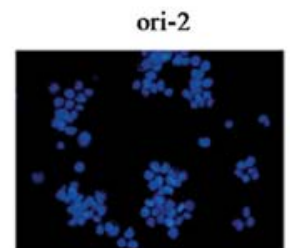

ori-16

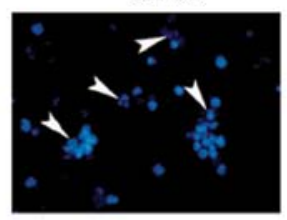

Figure 2. Apoptotic effects of oridonin on OCIM2. Oridonin-induced OCIM2 cell apoptosis in a dose-dependent manner. OCIM2 cells were treated with increasing doses of oridonin (0,2,8 and $16 \mu \mathrm{mol} / \mathrm{l})$ for $24 \mathrm{~h}$, and the apoptotic cells were measured by flow cytometry using (A) FACS and (B) Hoechst 33258 . Figures were representative of one of three experiments with similar results.

A

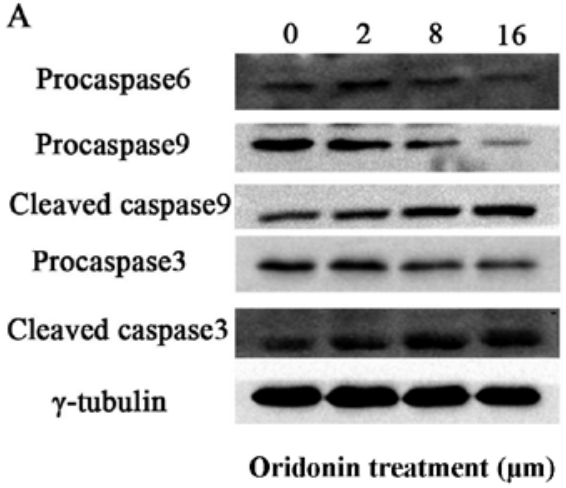

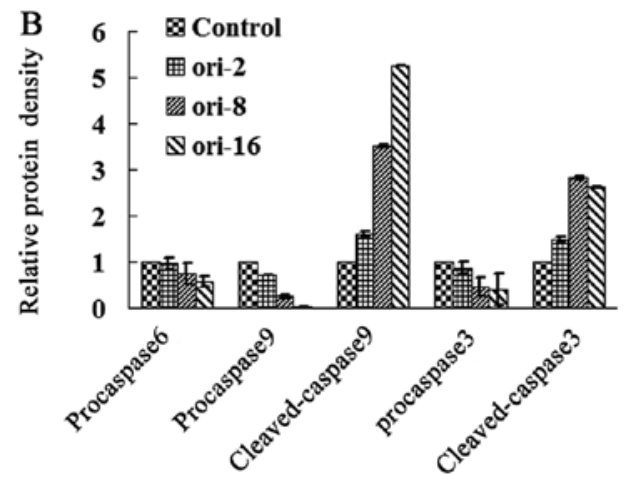

Figure 3. Activation of caspases by oridonin on OCIM2. (A) After cells were treated with different concentrations of oridonin ( $0,2,8$ and $16 \mu$ mol/l) for $24 \mathrm{~h}$, the whole cell lysates were subjected to $10 \%$ SDS-PAGE gel, electrotransferred on a nitrocellulose membrane and probed for the caspases-3, -6 and -9 . The same blots were stripped and reprobed with anti- $\gamma$-tubulin to show equal protein loading. Bands were quantified by densitometric analysis. (B) Level of the proteins was corrected relatively to the $\gamma$-tubulin and shown in the histogram. Figures were representative of one of three experiments with similar results.

the period of exposure increased, the $\mathrm{IC}_{50}$ values gradually decreased. The $\mathrm{IC}_{50}$ values in OCIM2 cells after 48 and $72 \mathrm{~h}$ were $10.12 \pm 1.24$ and $8.83 \pm 0.94 \mu \mathrm{mol} / 1$, respectively. The values were much lower $(3.18 \pm 0.42$ and $3.13 \pm 0.23 \mu \mathrm{mol} / \mathrm{l})$ in OCI-AML3 cells.

The anti-proliferative effects of oridonin on OCIM2 cells were also examined, using trypan dye exclusion. As shown in Fig. 1D, oridonin suppressed the vitality of OCIM2 within $48 \mathrm{~h}$, in a dose-dependent manner. These results showed that oridonin suppressed the proliferation of the tested AML cell lines, however, the oridonin concentration exerting its effect on the OCIM2 cells, was much higher compared to the concentration in OCI-AML3 cells.

Oridonin in a relatively high concentration induced OCIM2 cell-apoptosis. We investigated whether oridonin led to OCIM2 cell-apoptosis using the Annexin V/PI dual stain method. Annexin V binds to those cells that express phosphatidylserine on the outer layer of the cell membrane, a characteristic feature of cells entering apoptosis. To check this, OCIM2 cells were treated with different concentrations of oridonin for $24 \mathrm{~h}$ and then stained with Annexin V-FITC and PI. Results in Fig. 2A show a dose-dependent increase in Annexin V-positive cells, indicating the onset of apoptosis in oridonin-treated cells.

In addition, the effect of oridonin on OCIM2 cell apoptosis was observed subsequent to Hoechst 33258 staining, under laser scanning confocal microscope. Nuclei in the control cells were regular in shape. These cells decreased in size and darkened subsequent to oridonin treatment, while the nuclei presented chromatin condensation and marginalization or nuclear beading. The number of apoptotic cells increased with the concentration, while more typical morphological features appeared (8 and $16 \mu \mathrm{mol} / \mathrm{l})$ (Fig. 2B).

Oridonin-induced activated $N F-\kappa B$ was partially blocked in the cytoplasm of OCIM2 cells. Since studies have indicated $(4,5)$ that the $N F-\kappa B$ pathway was involved in oridonin-induced apoptosis, we examined whether or not $\mathrm{NF}-\kappa \mathrm{B}$ was activated by oridonin. OCIM2 cells were treated 
A

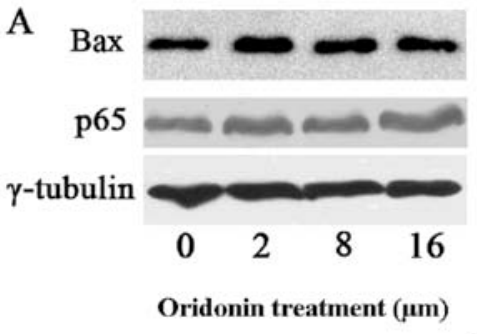

D

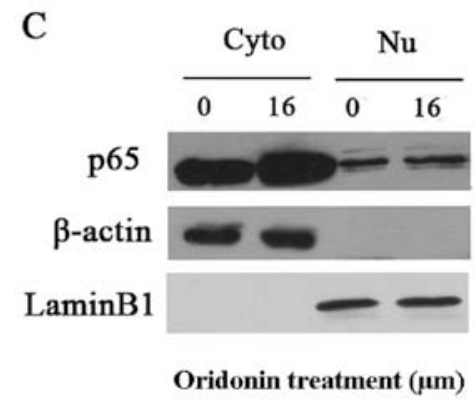

B
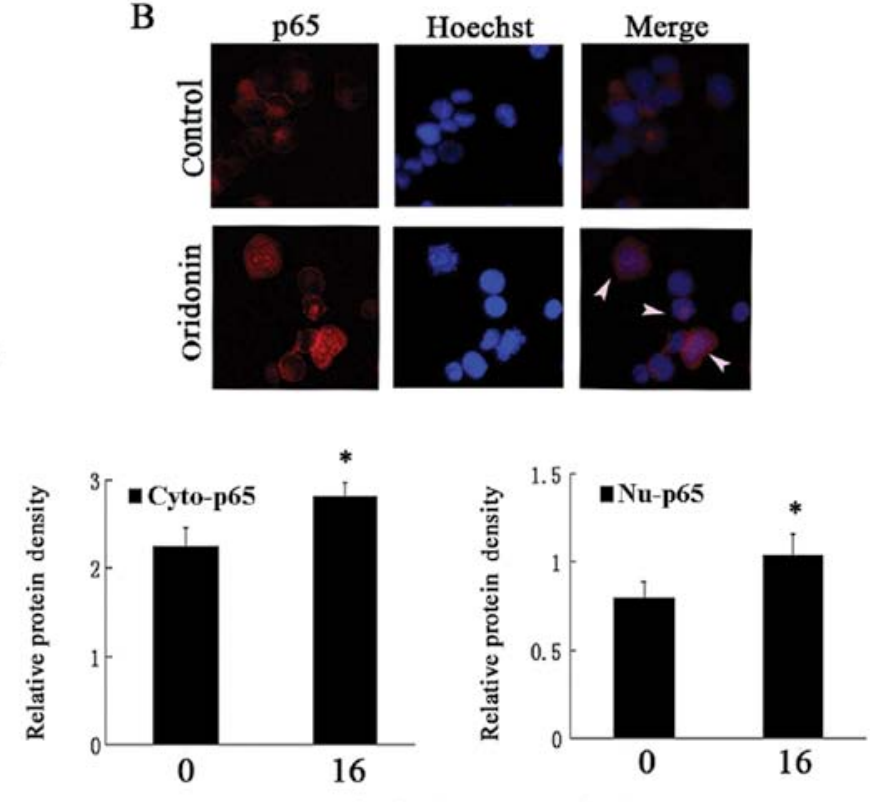

Oridonin treatment $(\mu \mathrm{m})$

Figure 4. Effects of oridonin on p65. Oridonin activated p65 and partly induced the translocation of p65 in OCIM2 cells. (A) OCIM2 cells were incubated with varying concentrations of oridonin for $24 \mathrm{~h}$, then the cell lysates were subjected to $10 \%$ SDS-PAGE gel, electrotransferred on a nitrocellulose membrane and probed for the p65 and Bax. The same blots were then stripped and reprobed with anti- $\gamma$-tubulin to show equal protein loading (lower panels). (B) OCIM2 cells were incubated alone or with oridonin for $24 \mathrm{~h}$ and then analyzed for the subcellular location of p 65 by immunofluorescence staining. Red stain indicated the localization of $\mathrm{p} 65$, and blue stain indicated nuclears (original magnification, $\mathrm{x} 200$ ). (C) OCIM2 cells were incubated along or with oridonin (16 $\mu \mathrm{mol} / \mathrm{l}$ ) for $24 \mathrm{~h}$, then the nuclear and cytosolic extracts were prepared and subjected to 10\% SDS-PAGE gel, electrotransferred on a nitrocellulose membrane and probed for the p65. The same blots were then stripped and reprobed with anti- $\beta$-actin and anti-laminB1 to show equal protein loading (lower panels). The bands were quantified by densitometric analysis. (D) The cytosolic and nuclear p65 were corrected to the $\beta$-actin and laminB1, respectively, and shown in the histogram. Figures were representative of one of three experiments with similar results. ${ }^{*} \mathrm{P}<0.05,{ }^{* *} \mathrm{P}<0.01$ vs. untreated control.

A

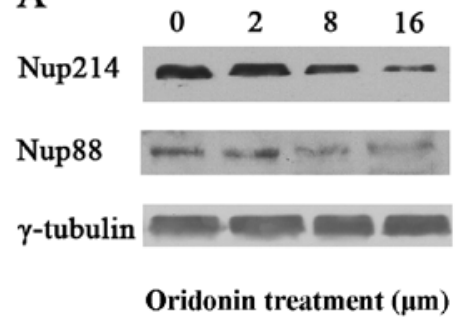

C

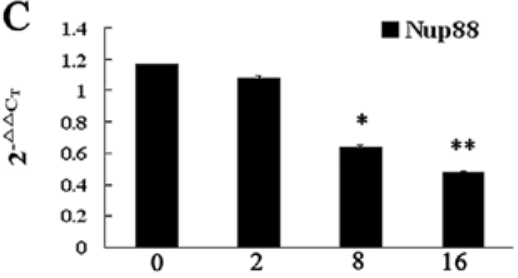

Oridonin treatment $(\mu \mathrm{m})$
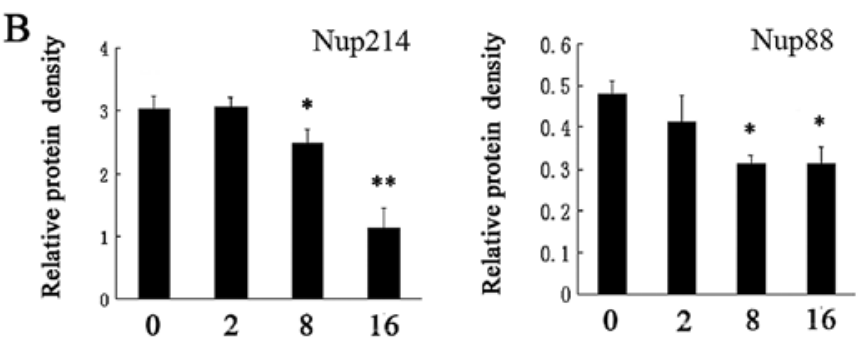

Oridonin treatment $(\mu \mathrm{m})$

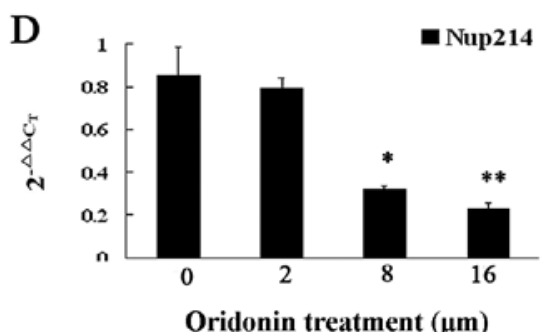

Oridonin treatment $(\mu \mathrm{m})$

Figure 5. Effects of oridonin on the Nup88 and Nup214. Oridonin downregulated Nup88 and Nup214 in the mRNA and protein levels. The OCIM2 cells were treated with the indicated doses of oridonin for $24 \mathrm{~h}$. (A) The protein level of the Nup88 and Nup214 in treated cells was detected by western blotting. Bands were quantified by densitometric analysis. (B) Nup88 and Nup214 were corrected relatively to the $\gamma$-tubulin and shown in the histogram. Nup88 and Nup214 mRNA of treated cells were detected by quantitative real-time RT-PCR. The histogram shows the $2^{-\Delta \Delta C T}$ values of (C) Nup88 and (D) Nup214. Data are presented as the mean $\pm \mathrm{SD}$ from three independent experiments. ${ }^{*} \mathrm{P}<0.05,{ }^{* *} \mathrm{P}<0.01$ vs. untreated control.

with varying concentrations of oridonin, while their protein extracts were monitored for p65 expression, using immunoblotting. Results in Fig. 4A show that the total p65 increased in a dose-dependent manner $(\mathrm{P}<0.05$ vs. untreated control).
While in the inactive state the $\mathrm{p} 65$ subunit was retained in the cytoplasm, in an activated NF- $\mathrm{\kappa B}$ the p65 subunit containing the transactivation domain was translocated into the nucleus. Therefore, to confirm that oridonin translocated p65 from the 
cytoplasm to the nucleus, immunofluorescence staining was used. The results in Fig. 4B clearly show that the p65 subunit of $\mathrm{NF}-\kappa \mathrm{B}$ translocated to the nucleus in the oridonin-treated group and especially in the typically apoptotic OCIM2 cells. In addition, subsequent to oridonin treatment the protein level of Bax increased accordingly (Fig. 4A). The downstream caspase effectors of Bax, such as caspases-9 and -3 were detected by western blot analysis. As shown in Fig. 3, oridonin significantly induced the activation of caspases- 9 and -3 while decreasing in the protein level of pro-caspases- 9 and -3 and increasing in the protein levels of cleaved forms in OCIM2 cells. In addition, oridonin treatment downregulated the protein level of pro-caspase-6, while indicating an increased immunofluorescence p65 intensity in the cytoplasm. To confirm a partially-retained p65 in the cytoplasm, the nuclear and cytosolic extracts of untreated and oridonin-treated cells were prepared and examined for p65 expression. Consistent with the results in Fig. 4B, results in Fig. 4C show that the cytoplasm p65 content increased in the oridonin-treated OCIM2 cells. As shown in Fig. 4D, there were statistically significant differences in p65 expression in the cytoplasm and nucleus in the oridonin-treated and control groups.

Partial translocation of p65 might be regulated by Nup 88 and Nup214. OCIM2 cells were markedly more resistant to oridonin compared to other AML cells, albeit oridonin had the potential to induce OCIM2 cell-apoptosis. In addition, p65 was observed to be retained in the cytoplasm of oridonin-treated OCIM2 cells, while the nucleocytoplasmic transport of p65 was reported to be selectively mediated by the Nup88/214 complex. We hypothesized that oridonin downregulated Nup88 as well as Nup214, while possibly inhibiting the translocation of p65 from the cytoplasm to the nucleus. To confirm the effect of oridonin on the Nup88 and Nup214 expressions, immunoblotting was employed on the OCIM2 cells treated with varying concentrations of oridonin as well as on their protein extracts, with a view to detect Nup88 and Nup214 expression. Results in Fig. 5A show that the Nup88 and Nup214 expressions decreased in a dosedependent manner.

To determine whether oridonin affected the transcriptional expression of Nup88 and Nup214, total RNA was extracted from untreated and oridonin-treated OCIM2 cells and Nup88, while Nup214 mRNA was analyzed by quantitative real-time PCR. Consistent with the results of immunoblotting, results shown in Fig. 5 demonstrate that the $2^{-\Delta \Delta \mathrm{CT}}$ of Nup88 (Fig. 5C) and Nup214 (Fig. 5D) were significantly decreased in the oridonin-treated, compared to the untreated cells.

\section{Discussion}

Oridonin, an active anti-cancer diterpenoid, is a well-known and widely used traditional Chinese medicament. Previous reports have demonstrated that oridonin had significant inhibitory effects on leukemia cells, including NB4, HL-60, U937, kausima-1, K562 and primary AML, both in vitro and in vivo (11-14). Although Zhou et al (12) found that oridonin-induced AML cell apoptosis $(8,21)$ through targeting the AML1-ETO (AE) fusion protein that is crucial in leuke- mogenesis, data regarding the potential of oridonin to induce apoptosis in erythroleukemia, as well as the precise underlying molecular mechanisms are scarce. In the present study, oridonin was found to have the potential to inhibit cell proliferation in the AML cell lines OCI-AML3 and OCIM2, when increasing treatment concentration. Subsequent to Hoechst 33258 staining, oridonin-treated OCIM2 cells demonstrated typical morphological changes, including nuclear chromatin condensation and a crescent shape. The number of apoptotic cells increased with the increased drug dose, while nuclei broke into fragments and formed apoptotic bodies. Flow cytometry-detected apoptosis showed a gradually increasing population of apoptotic cells. Based on these findings, a series of experiments were carried out to further determine potential apoptotic signaling pathways.

$N F-\kappa B$ regulates genes involved in cell survival, proliferation and apoptosis (15). Activation of NF- $\mathrm{NB}$ mediates cell survival signals in most tumor cells, acting simultaneously as a pro-apoptotic factor under certain conditions $(4,5)$. Results in the present study have shown that $N F-\kappa B$ was activated by oridonin in a dose-dependent manner, while the p65 translocated to the nucleus in the oridonin-treated group, especially in the typically apoptotic OCIM 2 cells, indicating that $\mathrm{NF}-\kappa \mathrm{B}$ promoted apoptosis in this study. Furthermore, oridonin treatment with varying concentrations was found to have upregulated the Bax expression, in accordance with the increasing $\mathrm{NF}-\kappa \mathrm{B}$ level.

Members of the Bcl-2 family, such as Bax, being one of the target genes of $N F-\kappa \mathrm{B}$ are crucial in the mitochondria pathway. The pro-apoptotic member Bax translocated to the mitochondria and integrated into the outer mitochondrial membrane, facilitating the release of cytochrome $c$ into the cytosol (16) and activating caspase-9, and subsequently cleaving and activating downstream effector proteases, such as caspase-3, resulting in apoptosis. In particular, caspase-3 has been shown to have a pivotal role in the terminal and execution phases of apoptosis induced by diverse stimuli (17). To elucidate whether Bax was involved in oridonininduced apoptosis, caspase- 9 expression and its downstream caspase were detected in oridonin-treated OCIM2 cells. The present study indicated that oridonin treatment with varying concentrations cleaved and activated caspase-9. Subsequently, caspase-3 was activated with an increase in the cleaved caspase- 3 protein, and a decrease in the protein levels of the pro-caspase-3 form. In addition, western blot analysis demonstrated that the pro-caspase- 6 decreased, thus oridonin activated caspase- 6 . However, most of the p65 was found to have been retained in the cytoplasm, possibly generating a markedly higher resistance to oridonin in OCIM2 cells.

Transport receptors recognizing nuclear import (NLS) or export signals (NES) on the cargo proteins are vital for the nucleocytoplasmic protein-transporting (6), while distinct nucleoporins determine substrate specificity. Previous reports identified anarrower specificity of Nup214 compared to CRM1-dependent export $(18,19)$. A study on Drosophila demonstrated that Drosophila members Only (mbo), an ortholog of Nup88, selectively regulated the nuclear translocation or export of dorsal, a member of the Rel protein family (20-22). Results in the present study showed that oridonin downregulated Nup214 and Nup88 at the gene and protein 
levels. As a result of the decreased Nup214 and Nup88, p65 did not translocate into the nucleus, since the nucleocytoplasmic transporting of p65 depended on Nup214 and Nup88. These results suggest that the downregulation of Nup214 and Nup88 protects OCIM2 cells by regulating the nucleocytoplasmic transport of $\mathrm{p} 65$.

\section{Acknowledgements}

This study was supported by a grant from the National Natural Science Foundation of China (nos. 30871036 and 81070429).

\section{References}

1. Santos FP, Bueso-Ramos CE and Ravandi F: Acute erythroleukemia: diagnosis and management. Expert Rev Hematol 3: 705-718, 2010.

2. Hayden MS and Ghosh S: Shared principles in NF-kappaB signaling. Cell 132: 344-362, 2008.

3. Ryan KM, Ernst MK, Rice NR and Vousden KH: Role of NF-kappaB in p53-mediated programmed cell death. Nature 404: 892-897, 2000

4. Baud V and Karin M: Is NF-kappaB a good target for cancer therapy? Hopes and pitfalls. Nat Rev Drug Discov 8: 33-40, 2009.

5. Perkins ND and Gilmore TD: Good cop, bad cop: the different faces of NF-kappaB. Cell Death Differ 13: 759-772, 2006.

6. Weis K: Nucleocytoplasmic transport: cargo trafficking across the border. Curr Opin Cell Biol 14: 328-335, 2002.

7. Gould VE, Orucevic A, Zentgraf H, Gattuso P, Martinez N and Alonso A: Nup88 (karyoporin) in human malignant neoplasms and dysplasias: correlations of immunostaining of tissue sections, cytologic smears, and immunoblot analysis. Hum Pathol 33: 536-544, 2002.

8. Gould VE, Martinez N, Orucevic A, Schneider J and Alonso A: A novel, nuclear pore-associated, widely distributed molecule overexpressed in oncogenesis and development. Am J Pathol 157: 1605-1613, 2000.

9. Emterling A, Skoglund J, Arbman G, et al: Clinicopathological significance of Nup88 expression in patients with colorectal cancer. Oncology 64: 361-369, 2003.
10. Fornerod M, Ohno M, Yoshida M and Mattaj IW: CRM1 is an export receptor for leucine-rich nuclear export signals. Cell 90: 1051-1060, 1997.

11. Gao F, Tang Q, Yang P, Fang Y, Li W and Wu Y: Apoptosis inducing and differentiation enhancement effect of oridonin on the all-trans-retinoic acid-sensitive and -resistant acute promyelocytic leukemia cells. Int J Lab Hematol 32: e114-e122, 2010.

12. Zhou GB, Chen SJ, Wang ZY and Chen Z: Back to the future of oridonin: again, compound from medicinal herb shows potent antileukemia efficacies in vitro and in vivo. Cell Res 17: 274-276, 2007.

13. Gao FH, Liu F, Wei W, et al: Oridonin induces apoptosis and senescence by increasing hydrogen peroxide and glutathione depletion in colorectal cancer cells. Int J Mol Med 29: 649-655, 2012.

14. Ji Z, Tang QJ, Zhang JS, Yang Y, Liu YF and Pan YJ: Oridonininduced apoptosis in SW620 human colorectal adenocarcinoma cells. Oncol Lett 2: 1303-1307, 2011.

15. Ghosh S and Karin M: Missing pieces in the NF-kappaB puzzle. Cell 109 (Suppl): S81-S96, 2002.

16. Kirkin V, Joos S and Zornig M: The role of Bcl-2 family members in tumorigenesis. Biochim Biophys Acta 1644: 229-249, 2004

17. Thornberry NA: Caspases: key mediators of apoptosis. Chem Biol 5: R97-R103, 1998

18. Bernad R, Engelsma D, Sanderson H, Pickersgill H and Fornerod M: Nup214-Nup88 nucleoporin subcomplex is required for CRM1-mediated $60 \mathrm{~S}$ preribosomal nuclear export. J Biol Chem 281: 19378-19386, 2006.

19. Hutten S and Kehlenbach RH: Nup214 is required for CRM1dependent nuclear protein export in vivo. Mol Cell Biol 26: 6772-6785, 2006.

20. Uv AE, Roth P, Xylourgidis N, Wickberg A, Cantera R and Samakovlis C: members only encodes a Drosophila nucleoporin required for rel protein import and immune response activation. Genes Dev 14: 1945-1957, 2000.

21. Roth P, Xylourgidis N, Sabri N, Uv A, Fornerod M and Samakovlis C: The Drosophila nucleoporin DNup88 localizes DNup214 and CRM1 on the nuclear envelope and attenuates NES-mediated nuclear export. J Cell Biol 163: 701-706, 2003.

22. Xylourgidis N, Roth P, Sabri N, Tsarouhas V and Samakovlis C: The nucleoporin Nup214 sequesters CRM1 at the nuclear rim and modulates NFkappaB activation in Drosophila. J Cell Sci 119: 4409-4419, 2006. 